\title{
Low-temperature positron transport in semi-insulating GaAs
}

\author{
Y. Y. Shan and K. G. Lynn \\ Department of Physics, Washington State University, Pullman, Washington 99164 \\ P. Asoka-Kumar \\ Physics Department, Brookhaven National Laboratory, Upton, New York 11973 \\ S. Fung and C. B. Beling \\ Department of Physics, The University of Hong Kong, Hong Kong
}

(Received 6 November 1996)

\begin{abstract}
Positron diffusion and drift in semi-insulating (SI) GaAs in the temperature range of 50-300 K were studied by the slow-positron beam technique. Both the temperature-dependent positron diffusion coefficient and positron mobility were measured independently using the method reported recently [Y. Y. Shan et al., Phys. Rev. B 54, 1982 (1996)]. The experimental results are consistent with the Einstein relation. The diffusion coefficient and mobility approximately follow $D_{+}(T)=9400 T^{-\beta} \mathrm{cm}^{2} \mathrm{~s}^{-1}$, and $\mu_{+}(T)=10^{8} \times T^{-\sigma} \mathrm{cm}^{2} \mathrm{~V}^{-1} \mathrm{~s}^{-1}$, with $\beta=1.5 \pm 0.1$, and $\sigma=2.5 \pm 0.2$, respectively in the temperature range of 50-300 K. The results are consistent with scattering from optical-phonon modes as the dominant scattering process for positron transport in GaAs (SI) in this temperature range. No trapped positron states were observed to $50 \mathrm{~K}$. [S0163-1829(97)00716-9]
\end{abstract}

\section{INTRODUCTION}

Carrier transport measurements are of interest for understanding the mechanisms of phonon and impurity interactions in solids. As a positive carrier of electric charge, the positron has a simpler band structure and relatively larger effective mass than electrons and holes in common semiconductors. ${ }^{1}$ The study of positron motion provides unique information about lattice scattering in solids, which is essential to many applications of the positron annihilation technique. $^{2}$

The positron motion can be characterized by measuring its diffusion coefficient $D_{+}$, and mobility $\mu_{+}$. There are several methods used to determine these two quantities. Mills and co-workers studied positron mobility by measuring its drift velocities based on the very small changes in the Doppler shift of the annihilation radiation. ${ }^{3,4}$ The positron lifetime method has been carried out to measure positron mobility in Si (Ref. 5) and GaAs. ${ }^{6}$ The positron beam technique has been effectively used to determine positron diffusion coefficients in metals ${ }^{7}$ and semiconductors. ${ }^{8-14}$ In these methods, either $D_{+}$or $\mu_{+}$can be obtained experimentally. If one is determined, the other is deduced via Einstein relation. So far, few measurements of independently determining both the positron mobility and diffusion coefficient have been reported.

GaAs, as an important semiconductor compound, has been extensively studied. The positron mobility value of $35 \pm 10$ and $60 \pm 20 \mathrm{~cm}^{2} \mathrm{~V}^{-1} \mathrm{~s}^{-1}$ at room temperature were reported by the drift velocity technique and lifetime technique, respectively, ${ }^{15,16}$ which, using the Einstein relation, relate to diffusion coefficients of $0.9 \pm 0.3$ and $1.6 \pm 0.5 \mathrm{~cm}^{2} \mathrm{~s}^{-1}$, respectively. Using the slow positron beam technique, diffusion lengths of $1800 \pm 140$ and $1500 \pm 100 \AA$ were reported for $n$-type $\mathrm{GaAs}$ at $300 \mathrm{~K}$, which correspond to positron diffusion coefficients of $0.9-$
$1.4 \mathrm{~cm}^{2} \mathrm{~s}^{-1} \cdot{ }^{17,18}$ Soininen et al. reported a diffusion coefficient of $1.6(2) \mathrm{cm}^{2} \mathrm{~s}^{-1}$ in semi-insulating (SI) GaAs at 300 K. ${ }^{15}$ More recently, a diffusion coefficient of 1.8(2) $\mathrm{cm}^{2} \mathrm{~s}^{-1}$ and a mobility of $70 \pm 10 \mathrm{~cm}^{2} \mathrm{~V}^{-1} \mathrm{~s}^{-1}$ in GaAs (SI) were obtained independently at room temperature. ${ }^{19}$

Measurements of the temperature effect on positron diffusion showed that scattering from acoustic phonons is predominant in cubic metals $(20-1400 \mathrm{~K}){ }^{7} \mathrm{Ge}(>500 \mathrm{~K}){ }^{14}$ and $\mathrm{Si}(30-500 \mathrm{~K}) .^{14,20,21}$ The diffusion coefficient follows the $T^{-1 / 2}$ power law. While in GaAs (SI), the diffusion coefficient exhibits a weak temperature dependence and does not follow the $T^{-1 / 2}$ power law in the temperature of 300$1000 \mathrm{~K} .{ }^{14}$ In $n$-type GaAs, clear deviations from $T^{-1 / 2}$ was observed in the temperature of $100-300 \mathrm{~K}^{18}$ It was attributed to positron shallow traps, possibly from negative compensating centers, where the positron is localized in Rydberg states.

In this study, monoenergetic positron beam measurements are provided for both the positron diffusion and drift in GaAs (SI) in the low-temperature range 50-300 K. The temperature dependence of the positron diffusion coefficient and mobility are independently determined. The results are consistent with the Einstein relation. The diffusion coefficient approximately follows the power law $D_{+} \propto T^{-3 / 2}$, indicating that positron motion is largely limited by optical-phonon scattering in this temperature range according to the calculation of the momentum relaxation time using the deformationpotential approximation. ${ }^{14,22-25}$

\section{EXPERIMENT}

The sample used in our experiments were undoped liquid-encapsulated-Czochralski-grown semi-insulating GaAs(100), grown by the ICI Wafer Technology Ltd. The room temperature resistivity of the sample was $10^{8} \Omega$, and the thickness was $0.5 \mathrm{~mm}$. The substrate was annealed at 
$500{ }^{\circ} \mathrm{C}$ for an hour to reduce possible positron trapping defects inside the bulk. This was confirmed by positron lifetime measurement in a previous work. ${ }^{26}$ It was then degreased in acetone and ethanol prior to being etched in standard solutions of $\mathrm{NH}_{4} \mathrm{OH}: \mathrm{H}_{2} \mathrm{O}_{2}: \mathrm{H}_{2} \mathrm{O}(3: 1: 90)$ and $\mathrm{H}_{2} \mathrm{SO}_{4}: \mathrm{H}_{2} \mathrm{O}_{2}: \mathrm{H}_{2} \mathrm{O}(8: 1: 1)$ for 1 min, respectively. A 1000 $\AA$ gold layer was evaporated onto each side of the sample to form a circular spot with 8-mm diameter. A dc bias was applied across the sample for the positron drift measurements.

Doppler broadening measurements of positron annihilation radiation were performed with a magnetically guided positron beam at Brookhaven National Laboratory. Fast positrons from a $50 \mathrm{~m} \mathrm{Ci}{ }^{22} \mathrm{Na}$ source was moderated in a 1$\mu \mathrm{m}$-thick $\mathrm{W}(100)$ single crystal foil in transmission geometry. The beam intensity was about $3 \times 10^{5} e^{+} / \mathrm{s}$, and its diameter was $4 \mathrm{~mm}$. The sample was mounted on the coldfinger of a closed-cycle $\mathrm{He}$ refrigerator cryostat for lowtemperature measurements and the pressure during the measurements was $10^{-8}$ mbar. The incident positron beam energy was varied from 0.1 to $50 \mathrm{keV}$. A high-purity $\mathrm{Ge}$ detector was used to detect the positron-electron annihilation gamma spectra. A total of $1 \times 10^{6}$ counts were collected under the 511-keV annihilation photopeak using a digitally stabilized multichannel analyzer.

\section{METHOD OF MEASURING BOTH TEMPERATURE-DEPENDENT POSITRON DIFFUSION COEFFICIENT AND MOBILITY}

Positrons thermalize rapidly ( $\sim 10 \mathrm{ps})$ after they are implanted into solids. ${ }^{27}$ Thermal positron transport can be modeled using the drift-diffusion equation. ${ }^{1,28}$ In our case, the system can be approximately treated as a semi-infinite space with a perfectly absorbing boundary at the $\mathrm{Au} / \mathrm{GaAs}$ interface. The drift-diffusion equation can be solved with proper boundary and initial conditions. ${ }^{19}$ By integrating the positron current density at the $\mathrm{Au} / \mathrm{GaAs}$ interface, the fraction of positrons reaching this interface $(z=0)$ can be obtained as ${ }^{19,29}$

$$
F_{\text {in }}=\int_{0}^{\infty} P_{E}(z) e^{-z / L_{\text {eff }}} d z,
$$

where $z$ denotes the distance from the interface along the positron beam entering direction. $P_{E}(z)$ is the implantation profile for a two layer structure, given as ${ }^{20,30-34}$

$$
P(z)=m \frac{(z+a)^{m-1}}{z_{0}^{m}} \exp \left[-\left(\frac{z+a}{z_{0}}\right)^{m}\right],
$$

where $a=\left(\rho_{\mathrm{Au}} / \rho_{\mathrm{GaAs}}\right) \Delta, \rho_{\mathrm{Au}}$ and $\rho_{\mathrm{GaAs}}$ are densities (in $\mathrm{g} \mathrm{cm}^{-3}$ ) of Au overlayer and GaAs crystal, respectively. $\Delta$ is the thickness of the Au layer. The parameters are taken as $\alpha=450 \AA \mathrm{g} \mathrm{cm}^{-3}, m=2.0, n=1.6 .^{32-36} z_{0}=$ $\left(\alpha / \rho_{\mathrm{GaAs}}\right) E^{n}$, and the beam energy $E$ is in $\mathrm{keV}$. The electric field is not expected to distort the implantation profile of the $\mathrm{keV}$ positrons. The positron mean implantation depth is $z_{0} \Gamma(1+1 / m)$.

The effective diffusion length

$$
L_{\text {eff }}(T, V)=\left\{\frac{\nu_{+}(V)}{2 D_{+}(T)}+\left[\frac{1}{D_{+} \tau}+\left(\frac{\nu_{+}}{2 D_{+}}\right)^{2}\right]^{1 / 2}\right\}^{-1}
$$

depends on both the temperature $T$ and the applied bias $V$ across the sample. $\nu_{+}$is the positron drift velocity, $D_{+}$is the positron diffusion coefficient, and $\tau$ is the positron lifetime. The measured line-shape parameter $S(E)$ can be expressed as $^{37}$

$$
S(E)=F_{\mathrm{Au}} S_{\mathrm{Au}}+\left(1-F_{\mathrm{Au}}\right)\left[F_{\text {in }} S_{\text {in }}+\left(1-F_{\text {in }}\right) S_{b}\right],
$$

where $F_{\mathrm{Au}}$ is the fraction of positrons annihilated in gold overlayer. $S_{\mathrm{Au}}, S_{\text {in }}$, and $S_{b}$ are the specific $S$ parameters of the Au overlayer, Au-GaAs interface, and bulk GaAs (SI). Thus an experimental value of the fraction of positrons reaching the absorbing boundary for beam energy $E$ at temperature $T$ under a bias of $V$ can be obtained as

$$
\begin{aligned}
F_{\text {in }}(E, T, V)= & \frac{1}{S_{b}(T)-S_{\mathrm{in}}(T)} \\
& \times\left[S_{b}(T)-\frac{S(E, T, V)-F_{\mathrm{Au}} S_{\mathrm{Au}}(T)}{1-F_{\mathrm{Au}}}\right] .
\end{aligned}
$$

When no bias is applied across the sample, i.e., $V=0$, the electric field throughout the sample is zero, expect in the narrow $\mathrm{Au} / \mathrm{GaAs}$ contact region where a small "built-in" contact potential exists. Since the "built-in" potential of $\mathrm{Au} / \mathrm{GaAs}$ is very small $(\sim 0.3 \mathrm{eV})$, this field effect in the contact region is neglected in our analysis and will not seriously influence the positron motion in the bulk. Under this approximation, from Eqs. (1) and (3), the fraction of positron diffusing back to the interface is

$$
F_{\text {in }}(E, T, 0)=\int_{0}^{\infty} P_{E}(z) e^{-z / L_{\text {eff }}(T, 0)} d z,
$$

where $L_{\text {eff }}(T, 0)=\left[D_{+}(T) \tau(T)\right]^{1 / 2}$. By fitting Eq. (6) to the experimental data, the temperature-dependent positron diffusion coefficient $D_{+}(T)$ can be obtained.

When a bias of $V$ is applied across the sample, the bias will drop mainly across the wide depletion region of the $\mathrm{Au} / \mathrm{GaAs}$ Schottky contact, creating a large electric field. The mean electric field in the depletion region is ${ }^{38}$

$$
E_{d}(V)=\left[\frac{e N_{D}\left(\phi_{\mathrm{bi}}+V-I R_{b}\right)}{2 \varepsilon_{0} \varepsilon_{r}}\right]^{1 / 2},
$$

where $I$ is the current flowing through the sample. $R_{b}$ is the bulk resistance. $\varepsilon_{r}$ is relative permittivity, and $N_{D}$ is the concentration of deep donor EL2 in GaAs (SI). $\phi_{\mathrm{bi}}$ is the "built-in" contact potential given by $e \phi_{\mathrm{bi}}=\phi_{b}-\left(E_{c}-E_{f}\right), \phi_{b}$ is the Schottky barrier height. $E_{c}-E_{f}$ is the bulk Fermi level measured from the bottom of the conduction band. Compared to the positron mean implantation depth of beam energy less than $50 \mathrm{keV}$ in this study, the wide depletion region $(\sim 3 \mu \mathrm{m})$ created by a large reverse bias $(\sim 25 \mathrm{~V})$ can be approximately treated as infinity. Thus the fraction of positron drift to the interface is

$$
F_{\text {in }}(E, T, V)=\int_{0}^{\infty} P_{E}(z) e^{-z / L_{\text {eff }}(T, V)} d z,
$$

where 


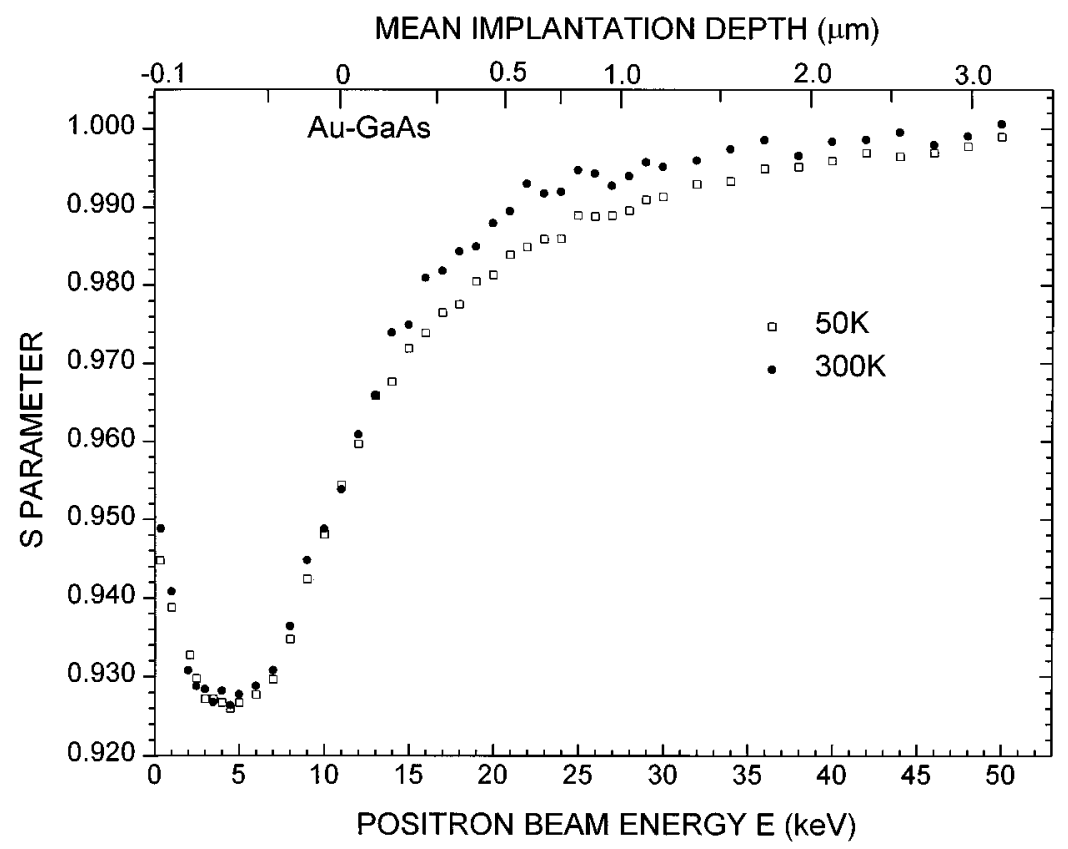

FIG. 1. The line-shape $S$ parameter as a function of the incident positron beam energy at temperatures 50 and $300 \mathrm{~K}$. The mean implantation depth for incident energies $E<11 \mathrm{keV}$ and $E \geqslant 11 \mathrm{keV}$, represents positron implantation mainly in the 1000 - $\AA$ overlayer and in bulk GaAs, respectively.

$L_{\text {eff }}(T, V)=\left\{\frac{\mu_{+} E_{d}(V)}{2 D_{+}(T)}+\left[\frac{1}{D_{+} \tau}+\left(\frac{\mu_{+} E_{d}}{2 D_{+}}\right)^{2}\right]^{1 / 2}\right\}^{-1}$.

By fitting Eq. (8) to the experimental data with a bias applied, a temperature-dependent positron mobility $\mu_{+}(T)$ can be obtained. Therefore, both temperature-dependent positron diffusion coefficient and mobility are determined experimentally. By comparing the obtained $D_{+}(T)$ and $\mu_{+}(T)$, a way to check the consistency of the Nerst-Einstein relation for positron transport in a wide temperature range can be provided.

\section{RESULTS AND DISCUSSIONS}

Figure 1 shows the measured $S$ parameter values as a function of positron incident energy at 50 and $300 \mathrm{~K}$. The $S$ values are normalized to the bulk GaAs $S$ value of 0.4985 .
The mean implantation depth is indicated on the upper axis. The smaller $S(E)$ values at $50 \mathrm{~K}$ indicate more positron diffusing to the $\mathrm{Au} / \mathrm{GaAs}$ interface at lower temperature because the interface $S$ value is smaller and the bulk $S$ value almost temperature independent, as presented in Fig. 2. The merge of the two $S(E)$ values at around $12 \mathrm{keV}$ indicates the $\mathrm{Au} / \mathrm{GaAs}$ is a perfect absorbing boundary, which means that all the positrons diffusing back to the interface are trapped and annihilate in the interface. This is in agreement with the lifetime measurements. ${ }^{26}$

Figure 2 shows the $S$ parameter as a function of temperature for several incident-beam energies. The measured $S$ value of $0.4985(5)$ at a beam energy of $50 \mathrm{keV}$ is assumed to be the bulk $S$ value of GaAs (SI), which shows a nearly temperature-independent behavior, and is in good agreement with other works. ${ }^{18,39}$ All the $S$ values are normalized to this bulk value. The constant $S$ value at $50 \mathrm{keV}$ indicates that no

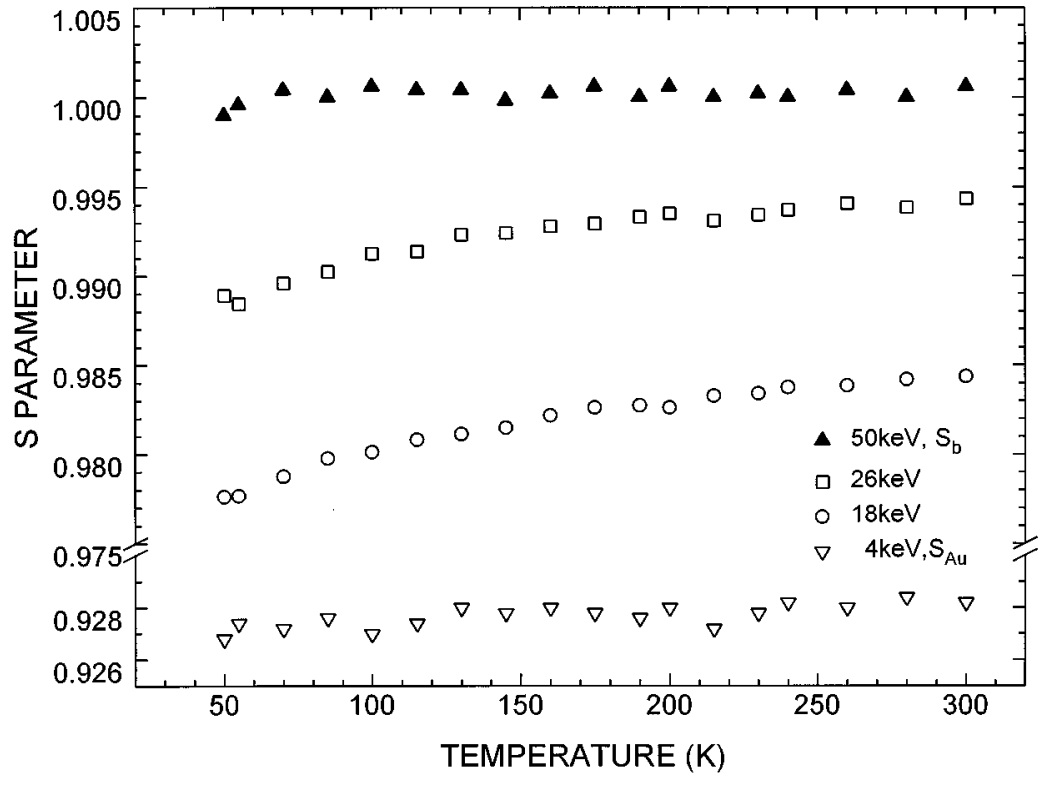

FIG. 2. Doppler broadening line-shape $S$ parameter as a function of sample temperature for incident positron energies of $4,18,26$, and 50 $\mathrm{keV}$, corresponding to the cases of positrons implanted meanly in the Au overlayer, in the region of $\mathrm{GaAs}$ close to $\mathrm{Au} / \mathrm{GaAs}$ interface, and in bulk GaAs. 


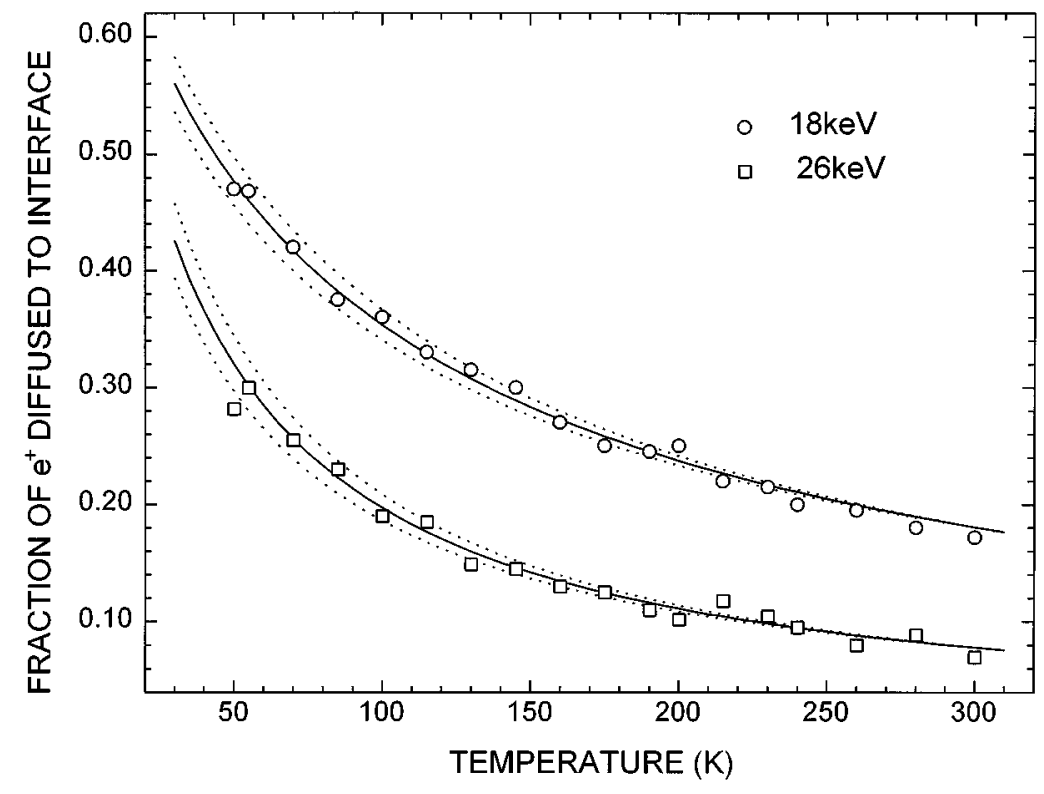

FIG. 3. The fraction of the positron diffusing back to the $\mathrm{Au} / \mathrm{GaAs}$ interface as a function of temperature for positron beam energies of 18 and $26 \mathrm{keV}$, respectively. The solid and dotted lines are the calculations from Eq. (8), corresponding to diffusion coefficient $D_{+}(T)=9400 T^{-\beta}$ $\mathrm{cm}^{2} \mathrm{~s}^{-1}, \beta=1.5 \pm 0.1$.

trapping at vacancy-type defects occurs in the studied temperature range. The $S$ value of $0.9265(5)$ for a beam energy of $4 \mathrm{keV}$ characterized the Au overlayer, which shows a very small linear increase possibly due to lattice expansion. So the specific $S$ values characterizing bulk GaAs $\left(S_{b}\right)$, Au/GaAs interface $\left(S_{\text {in }}\right)$, and Au overlayer $\left(S_{\mathrm{Au}}\right)$ were all assumed to be temperature independent, and were taken as 1.0, 0.9729, and 0.9265 , respectively, in our data analysis. The interface $S$ value can be obtained by fitting the $S-E$ curve. ${ }^{19}$ For incident energies of 18 and $26 \mathrm{keV}$, positrons are mainly implanted into the bulk region close to the interface. Consequently, a large amount of positrons will diffuse to the interface and annihilate there. Since diffusion is temperature dependent, the measured $S$ value as the function of temperature will characterize the temperature-dependent positron diffusion. When temperature decreases from 300 to $50 \mathrm{~K}, S$ values decrease from $0.9843(5)$ to $0.9775(5)$, and from $0.9944(5)$ to $0.9890(5)$ for beam energies of 18 and $26 \mathrm{keV}$, respectively. Although a measurement at a single energy value is sufficient to yield $D_{+}$and $\mu_{+}$, we used two beam energy values to obtain a more robust result.

Figure 3 shows the fractions of the positron $F_{\text {in }}$ diffusing back to the $\mathrm{Au} / \mathrm{GaAs}$ interface as a function of temperature. $F_{\text {in }}$ increases when the temperature decreases. By fitting Eq. (6) to the data, the temperature-dependent positron diffusivity $D_{+}$of $9400 \mathrm{~T}^{-\beta} \mathrm{cm}^{2} \mathrm{~s}^{-1}$, with $\beta=1.5 \pm 0.1$, was obtained. This result is consistent with the calculation of momentum relaxation time due to optical-phonon scattering using the deformation-potential approximation. ${ }^{14,22-25} \mathrm{Un}$ like cubic systems with only one atom in its primitive cell, zinc-blende lattice GaAs has two different atoms in its primitive cell. For each wave vector there are three acousticphonon modes and three optical-phonon modes. Opticalphonon scattering is important for positron diffusion in GaAs. As shown in earlier works, acoustic-phonon scattering alone could not explain the experimental results. ${ }^{14,15,18}$

In the above data analysis, the fraction of positron annihilated in the Au overlayer is calculated by

$$
F_{\mathrm{Au}}=\int_{-a}^{0} P_{E}(z) d z
$$

The implanted thermalized positrons in the evaporated $\mathrm{Au}$ overlayer can be assumed to be trapped, and to annihilate inside the $\mathrm{Au}$ layer where significant defects are present. Table I lists the values of the parameters used in the calculations.

When a reverse bias is applied across the sample, the bias drops mainly across the Au/GaAs Schottky barrier region, forming a wide depletion region with very strong electric field $\left(\sim 10^{5} \mathrm{~cm} \mathrm{~V}^{-1}\right)$. Positrons implanted into the depletion region will drift back to the $\mathrm{Au} / \mathrm{GaAs}$ interface. The drift effect depends on the positron mobility and the electric field it experiences. Figure 4 shows the $S$ parameter as a function of temperature under a reverse bias of $25 \mathrm{~V}$ for positron energies of 18 and $26 \mathrm{keV}$. Also, data for no bias are pre-

TABLE I. The values of the parameters used in the calculation fitting to the experimental data.

\begin{tabular}{lcc}
\hline \hline Relative permittivity of GaAs & $\varepsilon_{r}$ & $13.2^{\mathrm{a}}$ \\
Sample resistance at 300 K & $R^{b}$ & $9.95 \times 10^{6} \Omega$ \\
Thickness of the Au layer & $\Delta$ & $1000 \AA$ \\
Density & $\rho_{\mathrm{Au}}$ & $19.3 \mathrm{~g} \mathrm{~cm}^{-3}$ \\
& $\rho_{\mathrm{GaAs}}$ & $5.32 \mathrm{~g} \mathrm{~cm}^{-3}$ \\
Deep donor concentration & $N_{D}$ & $1.5 \times 10^{16 \mathrm{~cm}^{-3}}$ \\
Built-in potential & $\phi_{\mathrm{bi}}$ & $0.3 \mathrm{eV}^{\mathrm{b}}$ \\
Potential across the sample & $V$ & $25 \mathrm{~V}$ \\
& $\mathrm{IR}_{b}$ & $7.5 \mathrm{~V}$ \\
Implantation profile & $\alpha$ & $450 \AA \mathrm{g} \mathrm{cm}$ \\
& $m$ & 2.0 \\
Positron lifetime & $n$ & 1.6 \\
& $\tau(300 \mathrm{~K})$ & $230 \mathrm{ps}$ \\
\hline \hline
\end{tabular}

${ }^{\mathrm{a}}$ Sze (Ref. 38)

${ }^{\mathrm{b}}$ Rhoderick and Williams (Ref. 40).

${ }^{\mathrm{c}}$ Soininen et al. (Ref. 14). 


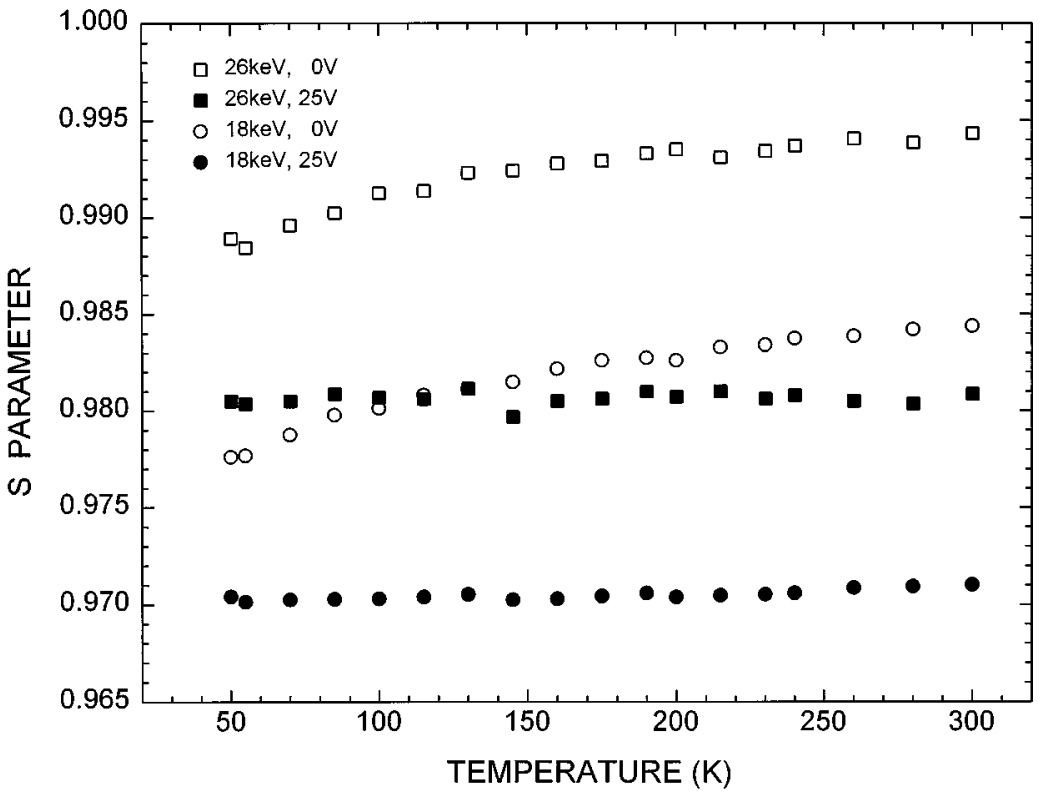

FIG. 4. The comparisons of zero-biased and 25-V reverse-biased line-shape $S$ parameters as a function of temperature for beam energies of 18 and $26 \mathrm{keV}$, respectively. sented for comparison. Smaller and less temperaturedependent $S$ values were obtained, comparing to those without bias. This can be understood because a large fraction of positrons implanted in the high-field depletion region will drift back to the interface of low- $S$ value when the contact is reverse biased. Since the electric field is very large $\left(\sim 10^{5} \mathrm{~cm} \mathrm{~V}^{-1}\right)$, saturation of positron drift can be easily achieved.

Figure 5 shows the fraction of positron drifting back to the interface as a function of temperature under a reverse bias of $25 \mathrm{~V}$ for beam energies of 18 and $26 \mathrm{keV}$, respectively. By fitting Eq. (8) to this experimental data, a positron mobility $\mu_{+}(T)$ of about $10^{8} T^{-\sigma} \mathrm{cm}^{2} \mathrm{~V}^{-1} \mathrm{~s}^{-1}$ with $\sigma=2.5 \pm 0.2$ was obtained. The electric field experienced by positron and the effective positron diffusion length were found by using Eqs. (7) and (9). The values of the parameters used in the calculation are listed in Table I. Because of the saturation in drift, $F_{\text {in }}$ cannot be enhanced much by decreas- ing the temperature if an appropriate reverse bias is applied. A possible high-efficiency positron moderator using GaAs (SI) can be operated at room temperature. ${ }^{16}$ On the other hand, $\sigma$ will not be sensitive to the above fitting when positron drifting is saturated. A more sensitive way of determining the temperature-dependent positron mobility is suggested. If an Ohmic metal-GaAs (SI) contact is prepared instead of a rectifying contact used in our measurements, a small and uniform electric field can be established across the sample. In such case, no saturation drift will happen, and a more accurate $\sigma$ value can be obtained. This may also be the way to improve positron mobility measurement using drift velocity method in which a uniform electric field is used. ${ }^{15}$

Figure 6 shows the consistency between the Einstein relation and the relation of the obtained positron diffusion coefficient and positron mobility in our measurements. The data points represent cases of the largest deviation from Einstein relation when $(\sigma-\beta)=1.0 \pm 0.3$ is obtained from the

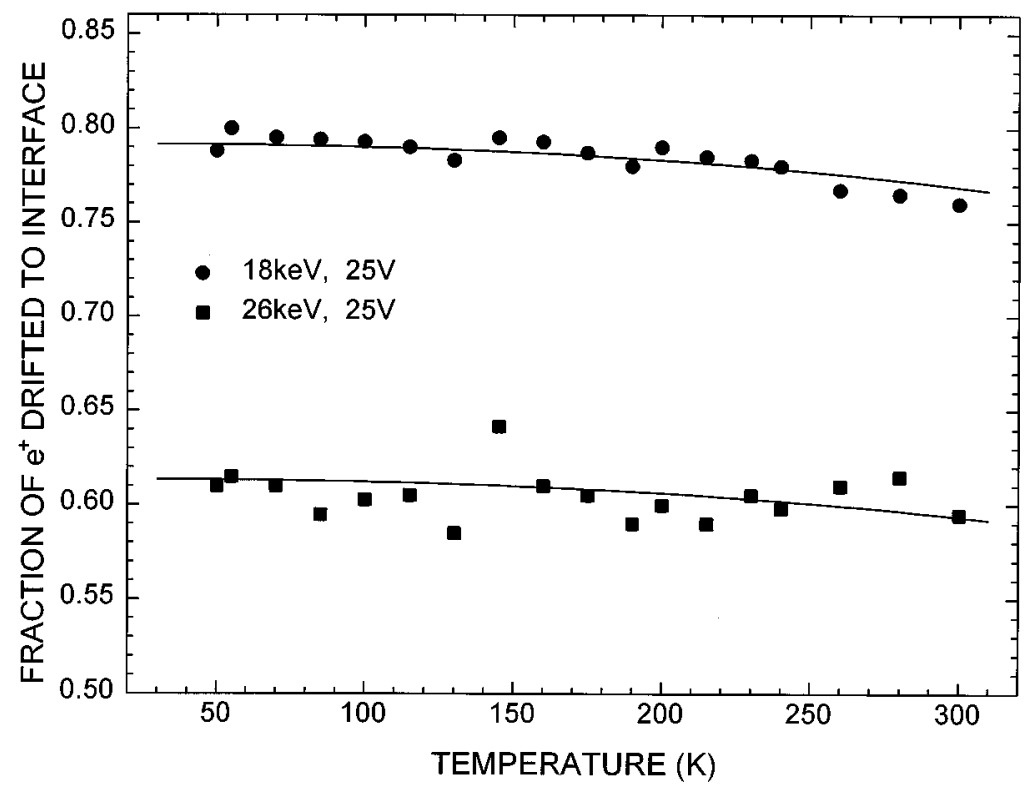

FIG. 5. The fraction of positrons drifting back to the $\mathrm{Au} / \mathrm{GaAs}$ interface under $25-\mathrm{V}$ reverse bias as a function of temperature with positron incident energies of 18 and $26 \mathrm{keV}$, respectively. The solid lines are the calculations from Eq. (10), with positron diffusion coefficient of $D_{+}(T)=9400 T^{-\beta} \mathrm{cm}^{2} \mathrm{~s}^{-1}, \beta=$ 1.5, and positron mobility of $\mu_{+}(T)=$ $10^{8} T^{-\sigma} \mathrm{cm}^{2} \mathrm{~V}^{-1} \mathrm{~s}^{-1}, \sigma=2.5$. 


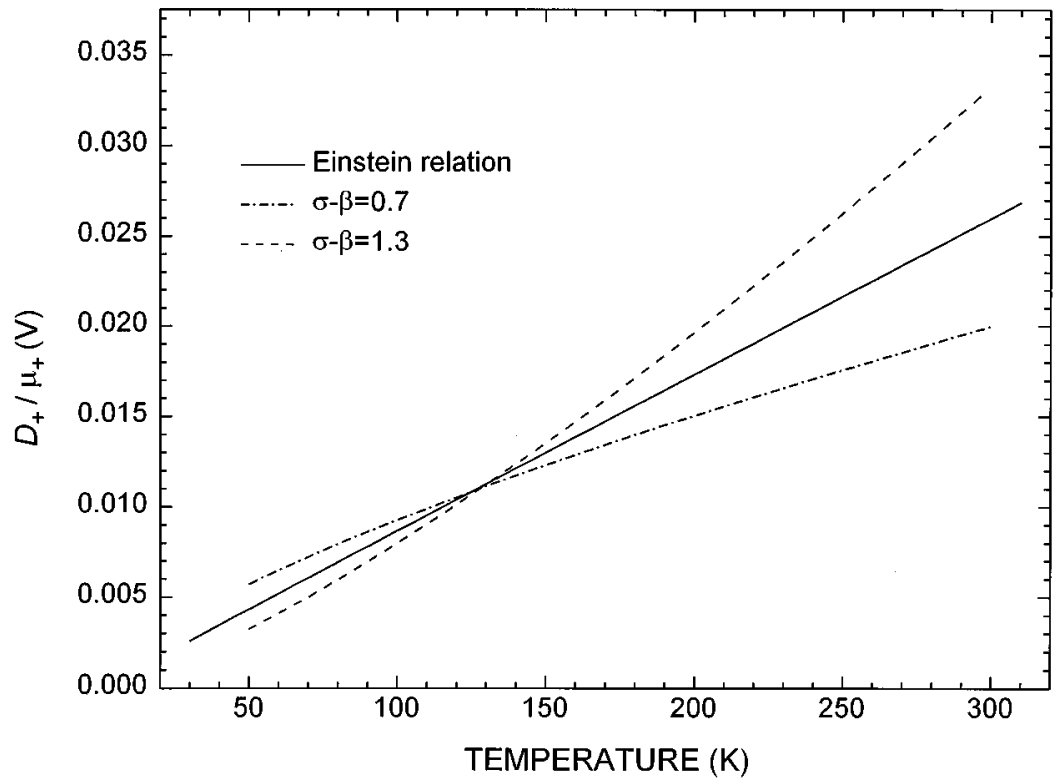

FIG. 6. Comparison between the experimental results of positron diffusion coefficient and mobility with the Einstein relation.

previous fitting shown in Figs. 3 and 5. The agreement in the low-temperature range supports that positron diffusion coefficient follows the $T^{-3 / 2}$ power law, i.e., the main scattering is from optical phonons in GaAs (SI) in the temperature range of $50-300 \mathrm{~K}$.

\section{CONCLUSIONS}

A monoenergetic positron beam of $0.1-50 \mathrm{keV}$ was used to study positron transport in semi-insulating GaAs in the temperature range of 50-300 K. Low-temperature effects on positron diffusion and drift were observed. The nearly temperature-independent bulk $S$ value is interpreted that no vacancy-type trap is present at low temperatures. Both temperature-dependent diffusion coefficient and mobility can be experimentally determined for positrons without resorting to the Einstein relation. A positron diffusion coefficient of
$9400 T^{-\beta} \mathrm{cm}^{2} \mathrm{~s}^{-1}$ and a positron mobility of $10^{8} T^{-\sigma}$ $\mathrm{cm}^{2} \mathrm{~V}^{-1} \mathrm{~s}^{-1}$ in GaAs (SI), with $\beta=1.5 \pm 0.1$ and $\sigma=2.5 \pm 0.2$, were obtained in the temperature range of 50$300 \mathrm{~K}$. A way to check the consistency of the Einstein relation for positron motion was provided in this work. The fact that the positron diffusion coefficient and mobility follow the above-temperature power law suggests optical-phonon scattering is the main scattering mechanism in GaAs (SI) in the temperature range studied.

\section{ACKNOWLEDGMENTS}

The authors wish to thank B. Nielsen, Cs. Szeles, and V. J. Ghosh for many valuable discussions and suggestions. This work was supported in part by the U.S. Department of Energy under Contract No. DE-AC02-76CH-00016.
${ }^{1}$ M. J. Puska and R. M. Nieminen, Rev. Mod. Phys. 66, 841 (1994).

${ }^{2}$ Positron Solid State Physics, edited by W. Brandt and A. Dupasquier (North-Holland, Amsterdam, 1983).

${ }^{3}$ A. P. Mills, Jr. and L. Pfeiffer, Phys. Rev. Lett. 36, 1389 (1976).

${ }^{4}$ A. P. Mills, Jr., E. M. Gulliksen, L. Pfeiffer, and W. S. Rockward, Phys. Rev. B 33, 7799 (1986).

${ }^{5}$ R. I. Simpson, M. G. Stewart, C. D. Beling, and M. Charlton, J. Phys. Condens. Matter 1, 7251 (1989).

${ }^{6}$ Y. Y. Shan, C. C. Ling, H. L. Au, S. Fung, C. D. Beling, and Y. Y. Wang, in Proceedings of the 10th International Conference on Positron Annihilation, Beijing, 1994, edited by Y. J. He, B. S. Cao, and Y. C. Jean, Mater. Sci. Forum Vols. 175-178 (Trans Tech, Aedermannsdorf, Switzerland, 1995), p. 517.

${ }^{7}$ E. Soininen, H. Huomo, P. A. Huttunen, J. Makinen, A. Vehanen, and P. Hautojärvi, Phys. Rev. B 41, 6227 (1990).

${ }^{8}$ P. J. Schultz, E. Tandberg, K. G. Lynn, B. Nielsen, T. E. Jackman, M. W. Denhoff, and G. C. Aers, Phys. Rev. Lett. 61, 187 (1988)
${ }^{9}$ B. Nielsen, K. G. Lynn, D. O. Welch, T. C. Leung, and G. W. Rubloff, Phys. Rev. B 40, 1434 (1989).

${ }^{10}$ A. P. Mills, Jr., Phys. Rev. Lett. 41, 1828 (1978).

${ }^{11}$ B. Nielsen, K. G. Lynn, A. Vehanen, and P. J. Schultz, Phys. Rev. B 32, 2296 (1985).

${ }^{12}$ H. H. Jorch, K. G. Lynn, and I. K. MacKenzie, Phys. Rev. Lett. 47, 362 (1981).

${ }^{13}$ H. H. Jorch, K. G. Lynn, and T. McMullen, Phys. Rev. B 30, 93 (1984).

${ }^{14}$ E. Soininen, J. Mäkinen, D. Beyer, and P. Hautojärvi, Phys. Rev. B 46, 13104 (1992).

${ }^{15}$ H. L. Au, C. C. Ling, T. C. Lee, C. D. Beling, and S. Fung, in Proceedings of the 9th International Conference on Positron Annihilation, edited by Z. Kajcsos and Cs. Szeles, Mater. Sci. Forum Vols. 105-111 (Trans Tech, Aedermannsdorf, Switzerland, 1992), p. 1363.

${ }^{16}$ Y. Y. Shan, H. L. Au, C. C. Ling, T. C. Lee, B. K. Panda, S. Fung, C. D. Beling, Y. Y. Wang, and H. M. Weng, Appl. Phys. A 59, 259 (1994). 
${ }^{17}$ P. C. Rice-Evans, D. L. Smith, H. E. Evans, and G. A. Gledhill, in Positron Beams for Solids and Surfaces, edited by P. J. Schultz, G. R. Massoumi, and P. J. Simpson, AIP Conference Proceedings No. 218 (AIP, New York, 1991), p. 147.

${ }^{18}$ K. Saarinen, P. Hautojärvi, A. Vehanen, R. Krause, and G. Dlubek, Phys. Rev. B 39, 5287 (1989).

${ }^{19}$ Y. Y. Shan, P. Asoka-Kumar, K. G. Lynn, S. Fung, and C. D. Beling, Phys. Rev. B 54, 1982 (1996).

${ }^{20}$ J. Mäkinen, C. Corbel, P. Hautojärvi, A. Vehanen, and D. Mathiot, Phys. Rev. B 42, 1750 (1990).

${ }^{21}$ J. Mäkinen, C. Corbel, P. Hautojärvi, and D. Mathiot, Phys. Rev. B 43, 12114 (1991).

${ }^{22}$ P. Lawaetz, Phys. Rev. 174, 867 (1968).

${ }^{23}$ E. M. Conwell, in High Field Transport in Semiconductors, Solid State Physics Supplement 9, edited by F. Seitz, D. Turnbull, and H. Ehrenreich (Academic, New York, 1967).

${ }^{24}$ B. Bergersen, E. Pajanne, P. Kubica, M. J. Stott, and C. H. Hodges, Solid State Commun. 15, 1377 (1974).

${ }^{25}$ B. K. Ridley, Quantum Processes in Semiconductors, 2nd ed. (Clarendon, Oxford, 1988).

${ }^{26}$ Y. Y. Shan, B. K. Panda, S. Fung, and C. D. Beling, Phys. Rev. B 52, 4724 (1995).
${ }^{27}$ Positrons in Solids, edited by P. Hautojärvi, Topics in Current Physics Vol. 12 (Springer, Berlin, 1979).

${ }^{28}$ P. J. Schultz and K. G. Lynn, Rev. Mod. Phys. 60, 701 (1988).

${ }^{29}$ A. P. Mills, Jr. and C. A. Murray, Appl. Phys. 21, 323 (1980).

${ }^{30}$ K. G. Lynn and D. O. Welch, Phys. Rev. B 22, 99 (1980).

${ }^{31}$ S. Valkealahti and R. M. Nieminen, Appl. Phys. A 32, 95 (1983).

${ }^{32}$ S. Valkealahti and R. M. Nieminen, Appl. Phys. A 35, 51 (1984).

${ }^{33}$ K. G. Lynn, D. M. Chen, B. Nielsen, and R. Pareja, Phys. Rev. B 34, 12114 (1986).

${ }^{34}$ A. Vehane, K. Saarinen, P. Hautojärvi, and H. Huomo, Phys. Rev. B 35, 4606 (1987).

${ }^{35}$ J. A. Baker, N. B. Chilton, and P. G. Coleman, Appl. Phys. Lett. 59, 164 (1991).

${ }^{36}$ P. Asoka-Kumar, K. G. Lynn, and D. O. Welch, J. Appl. Phys. 76, 4935 (1994).

${ }^{37}$ J. L. Campbell, Appl. Phys. 13, 365 (1977).

${ }^{38}$ S. M. Sze, Physics of Semiconductor Devices, 2nd ed. (Wiley, New York, 1981).

${ }^{39}$ R. Krause, K. Saarinen, P. Hautojärvi, A. Polity, G. Gärtner, and C. Corbel, Phys. Rev. Lett. 65, 3329 (1990).

${ }^{40}$ E. H. Rhoderick and R. H. Williams, Metal-Semiconductor Contacts, 2nd ed. (Clarendon, Oxford, 1988). 